\title{
Asociación entre la fuerza de las piernas y el área de sección muscular transversal del músculo cuádriceps femoral y el grado de actividad física en octogenarios
}

\author{
Pedro Á. Latorre-Román, Juan Manuel Arévalo-Arévalo, Felipe García-Pinillos \\ Departamento de Expresión Corporal, Universidad de Jaén, Jaén, España
}

\begin{abstract}
Introducción. El envejecimiento es un proceso fisiológico complejo cuya principal característica es la progresiva pérdida de funcionalidad, lo que es posible retardar o atenuar mejorando el estado físico. Objetivo. Analizar la asociación entre la fuerza de las piernas y el área de sección muscular transversal del músculo cuádriceps femoral, así como el nivel de actividad física en octogenarios.

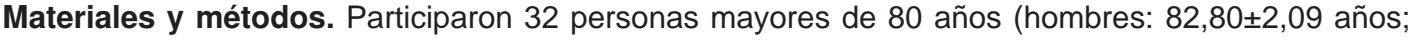

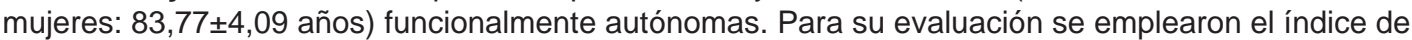
Barthel, el Yale Physical Activity Survey y la prueba de sentarse y levantarse.

Resultados. Se encontraron diferencias significativas entre sexos en la variable de área muscular $(p<0,001)$, en la prueba de sentarse y levantarse $(p=0,028)$ y el índice de paseo $(p=0,029)$, con valores superiores en los varones. El área muscular y la prueba de sentarse y levantarse se correlacionaron de manera significativa con el índice de paseo ( $r=0,445, p<0,005$ y $r=0,522, p<0,001$, respectivamente), y con el índice de actividad total semanal $(r=0,430, p<0,005$ y $r=0,519, p<0,001$, respectivamente). En el modelo de regresión lineal múltiple de este último índice, el área muscular y la prueba de sentarse y levantarse, solo esta última se comportó como variable predictora.

Conclusiones. La fuerza muscular y la masa muscular del músculo cuádriceps mostraron una asociación significativa con el grado de actividad física en personas mayores. La fuerza muscular de las piernas fue un indicador útil para revelar la masa muscular y el grado de actividad física en estas personas, lo cual tiene relevancia en la práctica clínica.
\end{abstract}

Palabras clave: anciano, índice de masa muscular, extremidad inferior, actividad motora. doi: http://dx.doi.org/10.7705/biomedica.v36i2.2654

Association between leg strength and muscle cross-sectional area of the quadriceps femoris with the physical activity level in octogenarians

Introduction: Aging is a complex physiological process whose main feature is the progressive loss of functionality, which may be delayed or attenuated by improving physical fitness.

Objective: To determine the association between leg strength and the muscle cross-sectional area of the quadriceps femoris in relation to physical activity level in the elderly.

Materials and methods: Thirty-two functionally autonomous people over 80 years (men: $82.80 \pm 2.09$ years; women: $83.77 \pm 4.09$ years) participated in this study. The Barthel Index, the Yale Physical Activity Survey and the Chair Stand Test were the instruments used.

Results: There were significant differences between sexes in muscle area $(p<0.001)$ in the Chair Stand Test $(p=0.028)$ and the walk index $(p=0.029)$, with higher values in men. The muscle area and the Chair Stand Test correlated significantly with the walk index $(r=0.445, p<0.005$, and $r=0.522, p<0.001$, respectively) and the total weekly activity index ( $r=0.430, p<0.005$, and $r=0.519, p<0.001$, respectively). In the multiple linear regression models for the total weekly activity index, muscle area and the Chair Stand Test, only the latter behaved as a predictor variable.

Conclusions: Muscle strength and muscle mass of quadriceps showed a significant association with the physical activity level in older people. Leg muscle strength was useful to reveal muscle mass and physical activity level in older people, which is relevant as a clinical practice indicator.

Key words: Aged, body mass index, lower extremity, motor activity.

doi: http://dx.doi.org/10.7705/biomedica.v36i2.2654

\section{Contribución de los autores:}

Pedro Á. Latorre-Román: diseño del estudio, análisis estadístico y redacción del artículo

Juan Manuel Arévalo-Arévalo: diseño del estudio y recolección de datos

Felipe García-Pinillos: redacción del artículo y recolección de datos 
El envejecimiento es un proceso fisiológico complejo cuya principal característica es la progresiva pérdida de funcionalidad, lo cual puede retardarse 0 atenuarse si se mejora la salud y el buen estado físico y mental, se evitan conductas de riesgo (consumo de tabaco, consumo excesivo de alcohol, exposición excesiva al sol y obesidad), se adopta la práctica habitual del ejercicio físico y se sigue una dieta saludable (1). Las limitaciones funcionales, definidas como la restricción de las capacidades físicas y mentales que se requieren para efectuar las actividades y tareas de una vida independiente, son importantes predictores de la mortalidad, la morbilidad y la discapacidad en el adulto mayor (2).

El envejecimiento se ha relacionado con la fragilidad y la limitación funcional debido a tres factores: el proceso biológico irreversible, el 'desacondicionamiento' debido al estilo de vida sedentario y los efectos de la comorbilidad (3). La reducción de la masa y la fuerza del músculo esquelético asociada con la edad, se define como sarcopenia (4). La disminución de la masa muscular es más prominente en los miembros inferiores (5).

Por otra parte, la producción de la fuerza máxima también disminuye con la edad y contribuye a la dependencia física y a la mortalidad (6). La dinapenia, término que también se utiliza para describir la pérdida de la fuerza muscular y la potencia relacionada con la edad (7), predispone a los adultos mayores a un mayor riesgo de limitaciones funcionales, discapacidad, morbilidad y mortalidad (7-10). Es probable que los déficits subclínicos en la estructura y la función del sistema nervioso y el deterioro en las propiedades de generación de fuerza del músculo esquelético sean antecedentes de la dinapenia (7).

En diversos estudios longitudinales se ha demostrado que a partir de los 75 años la fuerza se pierde a un ritmo de $3,4 \%$ anual en los hombres y de 2,5 a $3 \%$ en las mujeres; asimismo, los cambios en la fuerza muscular ocurren dos a cinco veces más rápidamente que la pérdida de masa, por lo tanto, la pérdida de fuerza es un mayor riesgo para la discapacidad y la muerte que la pérdida de masa

\footnotetext{
Correspondencia:

Pedro Á. Latorre-Román, Departamento de Expresión Corporal, Universidad de Jaén, Paraje Las Lagunillas $s / n$, edificio D2, Despacho 142, 23071, Jaén, España

Teléfono: (34) 953212710

platorre@ujaen.es

Recibido: 23/12/14; aceptado: 30/10/15
}

muscular (11). En una reciente revisión, Schaap, et al. (12), revelaron una importante asociación entre la fuerza muscular y la reducción de la funcionalidad en personas mayores. Se ha comprobado que la fuerza de extensión de la rodilla en relación con el peso corporal está asociada de forma relevante con la función física en personas de más de 75 años (13), específicamente, con la velocidad de la marcha (14). En este sentido, Takata, et al., han señalado la importancia de evaluar y conocer el estado de la condición física de las personas mayores, por ser un excelente indicador de la calidad de vida (15). En este caso, la evaluación de la fuerza de las piernas puede ser un factor que se debe tener en cuenta para determinar la funcionalidad y el grado de actividad física de las personas mayores.

En consecuencia, el objetivo de este estudio fue analizar la asociación de la fuerza de las piernas y el área muscular transversal de los cuádriceps femorales con el grado de actividad física en personas octogenarias.

\section{Materiales y métodos \\ Participantes}

En esta investigación participaron como voluntarios 32 sujetos (10 hombres y 22 mujeres) de una población de personas mayores de 80 años de ambos sexos (hombres: 82,80 $\pm 2,09$ años; mujeres: $83,77 \pm 4,09$ años), funcionalmente autónomas y residentes en centros para adultos mayores de la provincia de Jaén (España).

Los siguientes fueron los criterios de inclusión: personas octogenarias $\sin$ antecedentes de enfermedades metabólicas, insuficiencia cardiaca congestiva, dolores articulares, dolor en el pecho, vértigos, angina durante el ejercicio, o presión sanguínea alta no controlada, que no tuvieran marcapasos ni catéter, a quienes no se les hubiera desaconsejado el ejercicio físico por razones médicas, que no estuvieran tomando medicamentos con efectos sobre la fuerza muscular, que no presentaran deterioro cognitivo y no portaran prótesis metálicas implantadas quirúrgicamente.

\section{Instrumentos}

Para la valoración de la discapacidad física, se utilizó la versión validada en español del índice de Barthel (16), el cual mide de forma general el grado de independencia funcional del sujeto con respecto a la ejecución de algunas actividades básicas de la vida diaria, mediante puntuaciones de 0 a 100 en una escala de menor a mayor (17). 
Para la valoración del grado de actividad física, se emplearon los siguientes parámetros del Yale Physical Activity Survey en su versión en español (18): tiempo de actividad física, gasto energético semanal, índice de vigor, índice de paseo, índice de movimiento general, índice de estar de pie, índice de estar sentado e índice de actividad total semanal. La precisión, la validez y la reproducibilidad de este cuestionario aplicado en personas mayores son adecuadas $(19,20)$.

La fuerza muscular de las extremidades inferiores se analizó mediante la prueba de sentarse y levantarse durante 30 segundos (21), la cual implica sentarse y levantarse de una silla tantas veces como sea posible durante 30 segundos con los brazos cruzados sobre el pecho. El área de la sección transversal de masa muscular del músculo cuádriceps femoral (AMusc) se analizó mediante resonancia magnética (General Electric, $2421 \mathrm{~N}$ Mayfair Rd, Milwaukee, WI, USA).

\section{Procedimiento}

Los participantes fueron informados personalmente sobre el estudio en una primera reunión en los centros y residencias de la tercera edad en donde estaban internados. Se exceptuó a quienes no cumplían con los criterios de inclusión. Una vez reclutados, los sujetos firmaron el consentimiento informado y respondieron a los cuestionarios seleccionados, en los cuales se respetó el anonimato.

Posteriormente, se les practicaron una resonancia magnética y la prueba de fuerza de piernas, previa demostración de la forma de ejecutarla. Para la resonancia magnética, los participantes fueron examinados en posición supina, con las articulaciones de la rodilla y de la cadera extendidas y los brazos cruzados sobre el pecho. Se tomó una imagen situada en la mitad de la longitud del fémur, que comúnmente se utiliza para cuantificar la masa muscular del muslo, con cortes axiales de $5 \mathrm{~mm}$ contiguos a la espina ilíaca antero-superior en la línea de la articulación femorotibial de ambos muslos. Se identificaron dos puntos de referencia: $2 \mathrm{~cm}$ por debajo de la espina ilíaca antero-inferior (corte proximal) y la cara superior de la rótula (segmento distal). La distancia entre los sectores proximal y distal se dividió en partes iguales para seleccionar 17 cortes de la resonancia magnética. La masa muscular del cuádriceps femoral se determinó a partir de las mediciones hechas en estos 17 segmentos seleccionados.

\section{Análisis estadístico}

Los datos se analizaron con el programa estadístico SPSS ${ }^{\circledR}$, versión 19.0 para Windows (SPSS Inc., Chicago, USA). El nivel de significación (p) se fijó por debajo de 0,05 . Los datos se expresaron como media y desviación típica. Para la comparación de las diferentes variables por sexos, se empleó la prueba t de Student para muestras independientes. Se establecieron las correlaciones de Pearson para las variables analizadas y se hizo una regresión lineal múltiple por pasos hacia adelante utilizando como variable dependiente el índice de actividad total semanal.

\section{Consideraciones éticas}

Los participantes en esta investigación firmaron un consentimiento informado y los procedimientos experimentales se ajustaron a las normas éticas previstas en la Declaración de Helsinki (versión 2013), el Comité de Ética en Investigación con Seres Humanos de la Red Euro-Americana de Motricidad Humana y las directrices de la Comunidad Europea para la Buena Práctica Clínica (111/3976/88 de julio de 1990), así como el marco legal español para la investigación clínica en seres humanos (Real Decreto 561/1993 sobre ensayos clínicos). El consentimiento informado y el estudio fueron aprobados por el Comité de Bioética de la Universidad de Jaén, España.

\section{Resultados}

En el cuadro 1 se exponen los resultados de cada variable analizada según el sexo. Se puede observar que hubo diferencias significativas entre los sexos en las variables de AMusc $(p<0,001)$, en la prueba de sentarse y levantarse $(p=0,028)$, en el gasto energético semanal $(p=0,008)$ y en el índice de paseo $(p=0,029)$, con valores superiores en los varones.

En la figura 1 se muestra el área de sección transversal de masa muscular del cuádriceps femoral de dos varones, uno con 17 repeticiones en la prueba de sentarse y levantarse y otro con 11. Del mismo modo, en la figura 2 se muestran dos mujeres con diferentes resultados en la prueba de sentarse y levantarse, una con 19 repeticiones y otra con diez repeticiones. En las dos figuras se puede observar cómo las áreas de sección transversal del músculo cuádriceps del hombre y la mujer con más bajo rendimiento en la prueba fueron menores.

En el cuadro 2 se presentan las correlaciones de Pearson de las diferentes variables analizadas. Se puede destacar que el AMusc y los resultados de la 
Cuadro 1. Resultados de las variables analizadas por sexo

\begin{tabular}{lcrr}
\hline & $\begin{array}{c}\text { Hombres } \\
\text { Media (DT) }\end{array}$ & \multicolumn{1}{c}{$\begin{array}{c}\text { Mujeres } \\
\text { Media (DT) }\end{array}$} & $\mathbf{p}$ \\
\hline Edad & $82,80(2,09)$ & $83,77(4,09)$ & 0,486 \\
AMusc (mm $\left.{ }^{2}\right)$ & $13.544,28(1534,64)$ & $9.991,39(1613,71)$ & $<0,001$ \\
ChT (no repet.) & $12,50(6,00)$ & $8,27(4,165)$ & 0,028 \\
TActvF (h/sem) & $26,92(15,75)$ & $26,52(42,08)$ & 0,977 \\
IGT (Kcal/sem) & $6.116,10(4213,88)$ & $2.708,69(2529,72)$ & 0,008 \\
IV & $7,50(10,34)$ & $5,45(9,24)$ & 0,580 \\
IP & $24,80(15,41)$ & $12,73(12,98)$ & 0,029 \\
IM & $7,60(5,06)$ & $6,23(4,20)$ & 0,428 \\
IE & $5,40(3,40)$ & $4,45(2,30)$ & 0,363 \\
IS & $2,50(0,97)$ & $2,95(0,89)$ & 0,206 \\
IAT & $47,80(27,03)$ & $31,82(21,58)$ & 0,083 \\
IB $(0-100)$ & $99,00(3,16)$ & $95,23(10,05)$ & 0,259 \\
\hline
\end{tabular}

DT: desviación típica; AMusc: área muscular. ChT: prueba de sentarse y levantarse; TActvF: tiempo total de actividad física semanal; IGT: gasto energético semanal; IV: índice de vigor; IP: índice de paseo; IM: índice de movimiento general; IE: índice de estar de pie; IS: índice de estar sentado; IAT: índice de actividad total semanal; IB: índice de Barthel

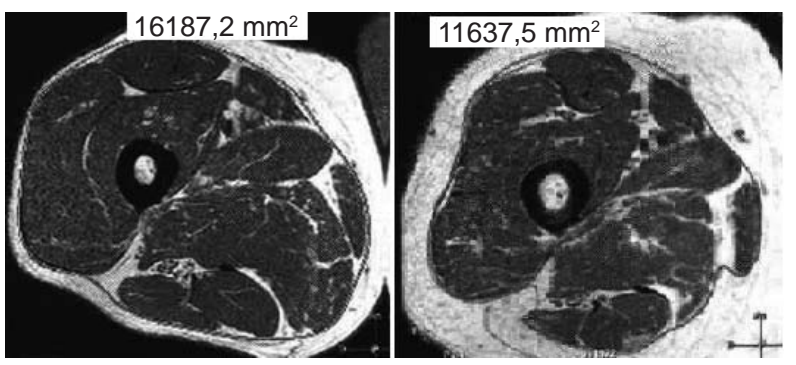

Figura 1. Área de la sección transversal de masa muscular del cuádriceps femoral de dos hombres; la figura de la izquierda corresponde a un hombre que ejecutó 17 repeticiones en la prueba de sentarse y levantarse y la figura de la derecha a otro que ejecutó 11 repeticiones en dicha prueba.

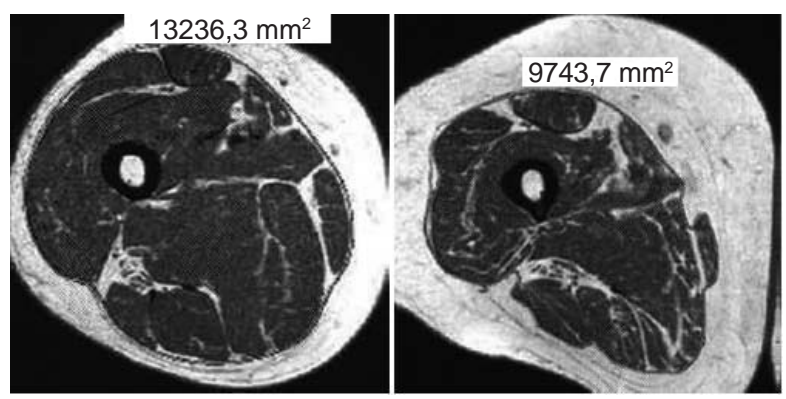

Figura 2. Área de la sección transversal de masa muscular del cuádriceps femoral de dos mujeres; la figura de la izquierda corresponde a una mujer que ejecutó 19 repeticiones en la prueba de sentarse y levantarse y la figura de la derecha a otra mujer que ejecutó diez repeticiones en dicha prueba.

prueba de sentarse y levantarse se correlacionaron de manera significativa con el índice de paseo $(r=0,445, p<0,05$ y $r=0,522, p<0,01$, respectivamente $)$ y el índice de actividad semanal total $(r=0,430$, $p<0,05$ y $r=0,519, p<0,01$, respectivamente).
En el modelo de regresión lineal múltiple de los resultados del índice de actividad total semanal, el AMusc y la prueba de sentarse y levantarse ajustados según el sexo, solo los de esta última se comportaron como variable predictora $(B=2,450$; $t=3,321 ; p=0,002)$. En la figura 3 se presenta el gráfico de dispersión de los resultados de la prueba de sentarse y levantarse y del índice de actividad semanal total, y se ve la asociación entre ellos tanto en hombres como en mujeres.

\section{Discusión}

El objetivo de este estudio fue analizar la asociación de la fuerza de las piernas y el área de sección muscular transversal de los cuádriceps femorales con el grado de actividad física en octogenarios. Como principal hallazgo se demostró que la prueba de sentarse y levantarse predijo el índice de actividad semanal total, pero no así el AMusc. Sin embargo, se registró una asociación de la prueba de sentarse y levantarse y el AMusc con el índice de paseo, el de actividad semanal total y el de Barthel. Estos resultados confirman la influencia del grado de actividad física total en la capacidad física, analizada en este caso mediante la prueba de sentarse y levantarse y la masa muscular.

En las personas de edad avanzada, la independencia funcional depende de la condición física y el envejecimiento se asocia con la disminución de las funciones de los sistemas y los órganos que determinan dicha condición (22). En este sentido, Bean, et al. (23), y Foldvari, et al. (24), han señalado la existencia de una asociación positiva entre la fuerza muscular y la capacidad de llevar a cabo actividades de la vida diaria, lo que puede ser 
Cuadro 2. Correlación de Pearson para las diferentes variables

\begin{tabular}{|c|c|c|c|c|c|c|c|c|c|c|c|c|}
\hline & Edad & AMusc & ChT & TActvF & IGT & IV & IP & IM & IE & IS & IAT & IB \\
\hline Edad & 1 & $-0,096$ & $-0,158$ & $-0,223$ & $-0,071$ & $-0,082$ & 0,135 & 0,043 & $-0,078$ & 0,008 & 0,049 & $-0,095$ \\
\hline AMusc & & 1 & $0,604^{* *}$ & 0,178 & $0,356^{*}$ & 0,265 & $0,445^{*}$ & 0,224 & 0,226 & $-0,279$ & $0,430^{*}$ & $0,475^{* *}$ \\
\hline ChT & & & 1 & $0,528^{* *}$ & 0,199 & 0,209 & $0,522^{* *}$ & $0,458^{* *}$ & $0,409^{*}$ & $-0,253$ & $0,519^{* *}$ & $0,484^{* *}$ \\
\hline TActvF & & & & 1 & 0,115 & 0,039 & $0,454^{* *}$ & $0,588^{* *}$ & $0,639^{*}$ & $-0,378^{*}$ & $0,455^{*}$ & 0,259 \\
\hline IGT & & & & & 1 & $0,501^{* *}$ & $0,468^{* *}$ & 0,262 & 0,333 & $-0,298$ & $0,554^{* *}$ & 0,348 \\
\hline IV & & & & & & 1 & 0,348 & 0,024 & 0,170 & $-0,250$ & $0,617^{* *}$ & 0,237 \\
\hline IP & & & & & & & 1 & $0,685^{* *}$ & $0,759^{* *}$ & $-0,531^{* *}$ & $0,934^{* *}$ & $0,412^{*}$ \\
\hline IM & & & & & & & & 1 & $0,791^{* *}$ & $-0,382^{*}$ & $0,683^{* *}$ & $0,353^{*}$ \\
\hline IE & & & & & & & & & 1 & $-0,485^{\star *}$ & $0,765^{* *}$ & $0,372^{*}$ \\
\hline IS & & & & & & & & & & 1 & $-0,506^{* *}$ & $-0,488^{* *}$ \\
\hline IAT & & & & & & & & & & & 1 & $0,430^{*}$ \\
\hline IB & & & & & & & & & & & . & 1 \\
\hline
\end{tabular}

AMusc: área muscular; ChT: prueba de sentarse y levantarse; TActvF: tiempo total de actividad física semanal; IGT: gasto energético semanal; IV: índice de vigor; IP: índice de paseo; IM: índice de movimiento general; IE: índice de estar de pie; IS: índice de estar sentado; IAT: índice de actividad total semanal; IB: índice de Barthel; * $p<0,05,{ }^{* *} p<0,01$

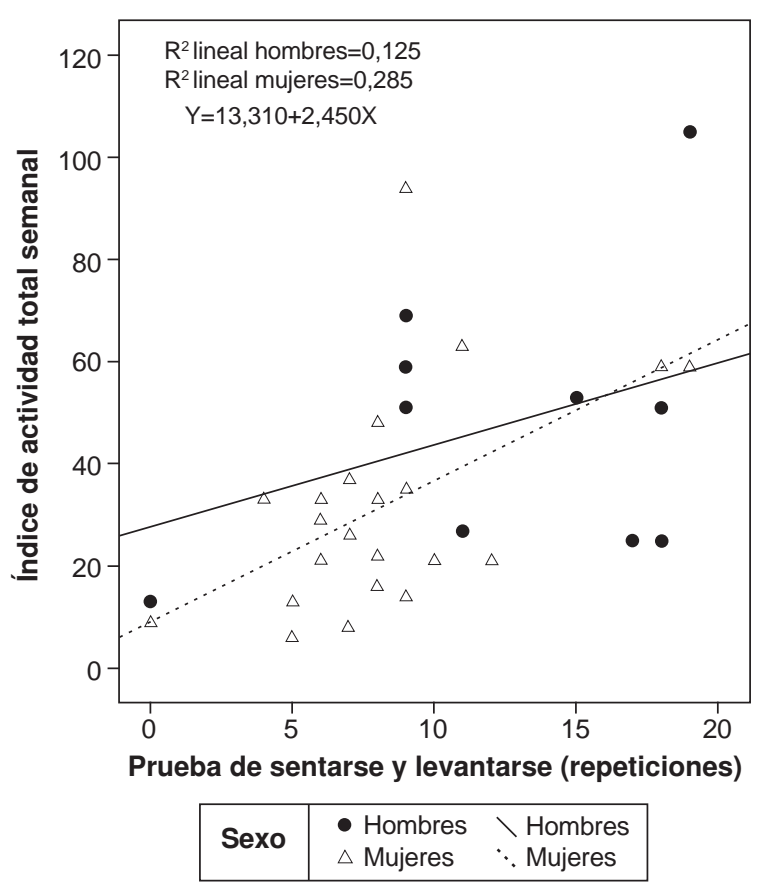

Figura 3. Diagrama de dispersión de la relación entre la variable de la prueba de sentarse y levantarse y el índice de actividad total semanal diferenciado por sexos

un predictor de la dependencia funcional $(25,26)$. Hairi, et al., registraron una reducción significativa de la fuerza del cuádriceps cada cinco años entre los 70 y los 90 años, y encontraron que la fuerza de las piernas constituía un importante descriptor de la limitación funcional (26). Del mismo modo, Bouchard, et al., han señalado que la fuerza de las piernas es un importante predictor de la función física en adultos mayores (27). En un estudio prospectivo con personas entre los 70 y los 79 años de edad, Visser, et al., demostraron que la masa muscular escasa (área de la sección transversal más pequeña del cuádriceps), una mayor infiltración de grasa en el músculo y una menor fuerza muscular extensora de la rodilla, se asociaban con un mayor riesgo de pérdida de la movilidad en los hombres y las mujeres de más edad (28). La asociación entre la escasa masa muscular y la disminución funcional parece ser una función de la fuerza muscular subyacente; sin embargo, la fuerza muscular plantea un mayor riesgo relativo para el desarrollo de la discapacidad que la escasa masa muscular (6).

Otro hallazgo relevante de este estudio fue que las mujeres mostraron un menor grado de actividad física y de fuerza de las piernas en comparación con los hombres, lo que concuerda con los resultados de Palacios, et al. (29). Sims, et al., reportaron en su estudio que la prevalencia de actividad física en los mayores de 65 años fue más elevada en hombres que en mujeres, y que las mujeres dedicaban menos tiempo a caminar que los hombres en todos los grupos de edad (30).

En este estudio el índice de paseo en las mujeres también fue más bajo que en los hombres. Esta disminución del grado de actividad física en mujeres mayores podría ser uno de los factores que explica la mayor prevalencia de dependencia funcional de las mujeres españolas ancianas en relación con los hombres: en España, 71,0 \% de las personas dependientes son mujeres y $45,7 \%$ de estas tiene 80 años de edad o más $(31,32)$. Aunque la masa muscular parece ser el principal determinante de las diferencias por edad y por sexo en la fuerza, la pérdida de fuerza muscular relacionada con la edad se explica por la reducción de la masa muscular solo en parte, pues otros factores neurológicos (por 
ejemplo, el reclutamiento de unidades motrices) y musculares (por ejemplo, la calidad contráctil y la infiltración de adipocitos), pueden desembocar en dinapenia.

Por lo tanto, mantener o ganar masa muscular no impide las caídas asociadas con el envejecimiento y la consecuente reducción de la fuerza muscular, y la relación de la debilidad muscular con la discapacidad física y la mortalidad es independiente de tales factores (7). Además, la actividad física puede ser una variable que interviene en la pérdida de fuerza muscular, de masa muscular (sarcopenia) y de calidad muscular (fuerza específica), en la limitación funcional y en la discapacidad física (26). En este sentido, en el estudio se registraron correlaciones significativas del índice de paseo y la actividad total semanal con el AMusc y la prueba de sentarse y levantarse.

Por último, cabe señalar que esta última prueba es un indicador accesible, sencillo y de bajo costo en la evaluación geriátrica. Su empleo facilita la detección de personas mayores con riesgo de dependencia funcional y la evaluación de los programas de intervención para la promoción de la autonomía personal en ancianos.

Una limitación del estudio fue que el análisis de la actividad física se basó en las respuestas a los cuestionarios y no en pruebas más objetivas como la acelerometría. Además, el carácter transversal del estudio obliga a ser cautos en las asociaciones encontradas entre las variables, por lo que se requieren estudios longitudinales y prospectivos para aclarar dichos hallazgos. Por otro lado, el empleo de la prueba de sentarse y levantarse durante 30 segundos para evaluar la fuerza debe considerarse como otra limitación del estudio, por lo cual el uso de métodos más precisos, como las máquinas isocinéticas, podría corroborar mejor los resultados. Asimismo, el hecho de que los sujetos estuviesen internados en instituciones restringe los resultados a este tipo de población.

En conclusión, se encontró una asociación significativa de la fuerza muscular y la masa muscular con el grado de actividad física en personas mayores, y la fuerza de las piernas resultó ser un indicador útil de su masa muscular y su grado de actividad física, lo cual tiene relevancia en la práctica clínica, especialmente en el seguimiento de la capacidad funcional de las personas mayores.

\section{Conflicto de intereses}

Los autores declaran no tener conflicto de intereses.

\section{Financiación}

No hubo fuentes de financiación externas.

\section{Referencias}

1. Castillo M, Ortega FB, Ruiz J. Mejora de la forma física como terapia antienvejecimiento. Med Clin. 2005;124:14655. http://dx.doi.org/10.1157/13071011

2. Reuben DB, Rubenstein LV, Hirsch SH. Value of functional status as a predictor of mortality: Results of a prospective study. Am J Med. 1992;93:663-9.

3. Rittweger J, Kwiet A, Felsenberg D. Physical performance in aging elite athletes challenging the limits of physiology. $J$ Musculoskelet Neuronal Interact. 2004;4:159-60.

4. Morley JE, Baumgartner RN, Roubenoff R, Mayer J, Nair KS. Sarcopenia. J Lab Clin Med. 2001;137:231-43. http:// dx.doi.org/10.1067/mlc.2001.113504

5. Janssen I, Heymsfield SB, Wang ZM, Ross R. Skeletal muscle mass and distribution in 468 men and women aged 18-88 yr. J Appl Physiol. 2000;89:81-8.

6. Clark BC, Manini TM. Maximal voluntary force (strength) production declines with age and contributes to physical dependence and mortality. J Gerontol A Biol Sci Med Sci. 2008;63:829-34.

7. Clark BC, Manini TM. What is dynapenia? Nutrition. 2012; 28:495-503. http://dx.doi.org/10.1016/j.nut.2011.12.002

8. Cruz-Jentoft AJ, Baeyens JP, Bauer JM, Boirie Y, Cederholm T, Landi F, et al. Sarcopenia: European Consensus on definition and diagnosis. Age Ageing. 2010;39:412-23. http://dx.doi.org/10.1093/ageing/afq034

9. Lauretani F, Russo CR, Bandinelli S, Bartali B, Cavazzini C, Di lorio A, et al. Age-associated changes in skeletal muscles and their effect on mobility: An operational diagnosis of sarcopenia. J Appl Physiol. 2003;95:1851-60. http://dx.doi.org/10.1152/japplphysiol.00246.2003

10. Metter EJ, Talbot LA, Schrager M, Conwit R. Skeletal muscle strength as a predictor of all-cause mortality in healthy men. J Gerontol. 2002;57:359-65. http://dx.doi. org/10.1093/gerona/57.10.B359

11. Mitchell WK, Williams J, Atherton P, Larvin M, Lund J, Narici M. Sarcopenia, dynapenia, and the impact of advancing age on human skeletal muscle size and strength; a quantitative review. Front Physiol. 2012;3:1-19. http:// dx.doi.org/10.3389/fphys.2012.00260

12. Schaap LA, Koster A, Visser M. Adiposity, muscle mass, and muscle strength in relation to functional decline in older persons. Epidemiol Rev. 2013;35:51-65. http://dx.doi. org/10.1093/epirev/mxs006

13. Barbat-Artigas S, Rolland Y, Cesari M, Abellan van Kan G, Vellas B, Aubertin-Leheudre M. Clinical relevance of different muscle strength indexes and functional impairment in women aged 75 years and older. J Gerontol A Biol Sci Med Sci. 2013;68:811-9. http://dx.doi.org/10.1093/gerona/ gls254

14. Hicks GE, Shardell M, Alley DE, Miller RR, Bandinelli S, Guralnik J, et al. Absolute strength and loss of strength as predictors of mobility decline in older adults: The InCHIANTI study. J Gerontol A Biol Sci Med Sci. 2012;67:66-73. http:// dx.doi.org/10.1093/gerona/glr055 
15. Takata Y, Ansai T, Soh I, Awano S, Yoshitake Y, Kimura $\mathbf{Y}$, et al. Quality of life and physical fitness in an 85-yearold population. Arch Gerontol Geriatr. 2010;50:272-6. http:// dx.doi.org/10.1016/j.archger.2009.04.005

16. Baztán JJ, Pérez del Molino J, Alarcón T, San Cristóbal E, Izquierdo G, Manzarbeitia J. Índice de Barthel, instrumento válido para la valoración funcional de pacientes con enfermedad cerebrovascular. Rev Esp Geriatr Gerontol. 1993;128:32-40.

17. Collin C, Wade DT, Davies S, Horne V. The Barthel ADL Index, a reliability study. Int Disabil Stud. 1988;10:61-3. http://dx.doi.org/10.3109/09638288809164103

18. De Abajo S, Larriba R, Márquez S. Validity and reliability of the Yale Physical Activity Survey in Spanish elderly. J Sports Med Phys Fitness. 2001;41:479-85.

19. Harada ND, Chiu V, King AC, Stewart AL. An evaluation of three self-report physical activity instruments for older adults. Med Sci Sports Exerc. 2001;33:962-70. http://dx.doi. org/10.1097/00005768-200106000-00016

20. Schuler PB, Richardson MT, Ochoa P, Wang MQ. Accuracy and repeatability of the Yale Physical Activity Survey in assessing physical activity of older adults. Percept Mot Skills. 2001;93:163-77. http://dx.doi.org/10.2466/pms. 2001.93.1.163

21. Jones CJ, Rikli RE, Beam WC. A 30-s chair-stand test as a measure of lower body strength in community-residing older adults. Res Q Exerc Sport. 1999;70:113-9. http://dx.doi.org/ 10.1080/02701367.1999.10608028

22. Garatachea N, Lucía A. Genes, physical fitness and ageing. Ageing Res Rev. 2013;12:90-102. http://dx.doi. org/10.1016/j.arr.2012.09.003

23. Bean JF, Leveille SG, Kiely DK, Bandinelli S, Guralnik JM, Ferrucci L. A comparison of leg power and leg strength within the InCHIANTI study: Which influences mobility more? J Gerontol A Biol Sci Med Sci. 2003;58:728-33. http:// dx.doi.org/10.1093/gerona/58.8.M728

24. Foldvari M, Clark M, Laviolette LC, Bernstein MA, Kaliton D, Castañeda C, et al. Association of muscle power with functional status in community dwelling elderly women. J Gerontol A Biol Sci Med Sci. 2000;55:192-9. http://dx.doi. org/10.1093/gerona/55.4.M192
25. Yoon BK, Kim CH, Lim HJ, Kim Y, Im J, Paik II, et al. Association of physical performance and health-related factors among elderly Koreans. International Sport Med Journal. 2009;10:205-15.

26. Hairi NN, Cumming RG, Naganathan V, Handelsman DJ, Le Couteur DG, Creasey $\mathrm{H}$, et al. Loss of muscle strength, mass (sarcopenia), and quality (specific force) and its relationship with functional limitation and physical disability: The Concord Health and Ageing in Men Project. J Am Geriatr Soc. 2010;58:2055-62. http://dx.doi.org/10.1111/ j.1532-5415.2010.03145.x

27. Bouchard DR, Heroux M, Janssen I. Association between muscle mass, leg strength, and fat mass with physical function in older adults: Influence of age and sex. J Aging Health. 2011;23:313-28. http://dx.doi.org/10. $1177 / 0898264310388562$

28. Visser M, Goodpaster BH, Kritchevsky SB, Newman AB, Nevitt M, Rubin SM, et al. Muscle mass, muscle strength, and muscle fat infiltration as predictors of incident mobility limitations in well-functioning older persons. J Gerontol A Biol. 2005;60:324-33. http://dx.doi.org/10.1093/ gerona/60.3.324

29. Palacios-Ceña D, Alonso-Blanco $\mathbf{C}$, Jiménez-García R, Hernández-Barrera V, Carrasco-Garrido P, PileñoMartínez E, et al. Time trends in leisure time physical activity and physical fitness in elderly people: 20 year follow-up of the Spanish population national health survey (1987-2006). BMC Public Health. 2011;11:799. http://dx.doi. org/10.1186/1471-2458-11-799

30. Sims J, Birrell CL, Hunt S, Browning C, Burns RA, Mitchell P. Prevalence of physical activity behaviour in older people: Findings from the Dynamic Analyses to Optimise Ageing (DYNOPTA) project and Australian national survey data. Australas J Ageing. 2014;33:105-13. http://dx.doi. org/10.1111/j.1741-6612.2012.00648.x

31. Abellán-García A, Esparza-Catalán C, Pérez-Díaz J. Evolución y estructura de la población en situación de dependencia. Cuad Relac Laborales. 2011;29:93-123.

32. Abellán A, Esparza C, Castejón P, Pérez J. Epidemiología de la discapacidad y la dependencia de la vejez en España. Gaceta Sanitaria. 2011; 25:5-11. 